\title{
Trends in Lipids and Lipoproteins Among Adults in Northwestern Xinjiang, China, From 1998 Through 2015
}

Ling Zhou*, Xin Zhao*, Mulalibieke Heizhati, Suofeiya Abulikemu, Delian Zhang, Qiuyan Cheng, Weijin Ouyang, Xiaoguang Yao, Jing Hong, Ting Wu, Zuhere Xiamili, and Nanfang Li

The Center of Hypertension of the People's Hospital of Xinjiang Uygur Autonomous Region, The Hypertension Institute of Xinjiang, Xinjiang, China

Received February 15, 2018; accepted June 14, 2018; released online December 22, 2018

\begin{abstract}
Background: To examine trends in serum lipids in population in Northwestern Xinjiang between 1998 and 2015 and to provide clues for future prevention

Methods: We enrolled 5,142 adults aged $\geq 30$ years from seven independent cross-sectional studies conducted in 1998-2000, 2007-2008, and 2015. Blood lipid profiles, such as total cholesterol (TC), triglyceride (TG), high-density lipoprotein cholesterol (HDL-C), and low-density lipoprotein cholesterol (LDL-C), were measured.

Results: The mean age was 48.5 years in 1998-2000, 47.9 years in 2007-2008, and 53.7 years in 2015. There was a declining trend in the prevalence of dyslipidemia among adults in northwestern Xinjiang. Mean LDL-C decreased during the same period, while mean HDL-C showed the opposite trend. Mean TC was $4.79 \mathrm{mmol} / \mathrm{L}$ in 1998-2000, $5.17 \mathrm{mmol} / \mathrm{L}$ in 2007-2008, and $4.59 \mathrm{mmol} / \mathrm{L}$ in 2015 . The trend of mean TG was similar to that of TC. The prevalence of dyslipidemia was closely related with male gender, Mongolian ethnicity, hypertension, obesity, elevated fasting blood glucose, smoking, and drinking.

Conclusion: Between 1998 and 2015, favorable trends in lipid levels have occurred among adults of Northwestern Xinjiang. However, further efforts are needed.
\end{abstract}

Key words: lipid; dyslipidemia; ethnicity; epidemiologic study; risk factors

Copyright $\odot 2018$ Ling Zhou et al. This is an open access article distributed under the terms of Creative Commons Attribution License, which permits unrestricted use, distribution, and reproduction in any medium, provided the original author and source are credited.

\section{INTRODUCTION}

Cardiovascular disease (CVD) is the leading cause of death worldwide and accounts for $31 \%$ of all global deaths. ${ }^{1}$ The World Health Organization (WHO) reported that, about 17.7 million people died from CVD worldwide in 2015. ${ }^{1}$ Epidemiologic surveys have demonstrated that high concentrations of total cholesterol (TC) and low-density lipoprotein cholesterol (LDL-C) and low levels of high-density lipoprotein cholesterol (HDL-C) are major risk factors for CVD. ${ }^{2-8}$ Many previous studies have proved that mortality and morbidity from CVD can be reduced by lowering the serum lipids levels. , $^{3,9-12}$

Over 28 years, mean TC has decreased from high levels in the high-income western countries, whereas it increased in east and southeast Asia and Pacific by $0.08 \mathrm{mmol} / \mathrm{L}$ per decade in males and $0.09 \mathrm{mmol} / \mathrm{L}$ per decade in females. ${ }^{13}$ In China, the prevalence of dyslipidemia in adults has increased from $18.6 \%$ in 2002 to $40.1 \%$ in $2012 .{ }^{14,15}$ Elevated serum cholesterol levels are expected to result in 9.2 million extra CVD events in China during the next 20 years. ${ }^{16}$

Xinjiang, a vast and multi-ethnic province, is located in the northwest edge of China. It is the largest Chinese administrative region and takes up about one-sixth of the country's territory. Their major employment is peasants or herdsmen in this agricultural and pastoral area. Previous studies have shown that the prevalence of dyslipidemia in Xinjiang is higher than the national level. ${ }^{17-20}$ The prevalence of dyslipidemia in Xinjiang was $49.2 \%$ in $1997-1998 .^{19}$

This study observed that significant differences also existed in lipid levels and prevalence of dyslipidemia among Kazakh (53.5\%), Mongolian (49.3\%), and Han (47.3\%) ethnic groups, which is likely to be caused by different lifestyle and diet-related habits. Earlier studies have indicated that Kazakhs consume more animal meat and fewer vegetables. ${ }^{21}$ Historically, there was a drastic difference in the living environment between the ethnic groups. Most Kazakhs and Mongolians reside in stock-raising areas like villages, forests, and mountains and move around with seasonal changes, which makes their living depend much on animal products, including animal oil, meat, milk, and dairy products and makes it difficult to have enough intake of vegetables and fruits. Furthermore, some roles of genetics have been described, ${ }^{22}$ although not confirmed. Meanwhile, Xinjiang, previously had lower education attainment (illiteracy rate: about $11.44 \%$, higher than the national level of $9.08 \%$ in 2000$),{ }^{23}$ relatively backward economy, and extremely immature medical conditions, which makes it difficult to implement health education. Nonetheless, in recent years, socio-economic conditions, education attainment, and health care resources have

Address for correspondence. Nanfang Li, The Center of Hypertension of the People's Hospital of Xinjiang Uygur Autonomous Region, The Hypertension Institute of Xinjiang, No. 91 Tianchi Road Tianshan District, Urumqi 830001, Xinjiang, China (e-mail: lnanfang2016@sina.com).

*These authors equally contributed to this work. 
shown significant development in Xinjiang. The Gross domestic product increased from 111.66 billion RMB in 1998 to 932.48 billion RMB in $2015 .^{24}$ The proportion of population acquiring senior middle or higher education increased from $19.0 \%$ $(n=3,194,374)$ in 2000 to $24.3 \%(n=4,860,941)$ in $2010 . .^{23,25}$ Moreover, increases in coverage and utilization of health care resources, particularly the implementation of new rural medical cooperative care system, were seen in China since 2003. ${ }^{26}$ Therefore, it is reasonable to believe that all the changes over these years might have had beneficial effects on lipid levels and on the prevalence of dyslipidemia. However, there have been no subsequent updates of the prevalence of dyslipidemia and trends in lipid profiles in this area.

Therefore, this study aimed to examine recent trends in lipid profiles, including lipid ratios and the prevalence of dyslipidemia, in three counties of northwestern Xinjiang Province based on seven independent population surveys performed in Hefeng (1998, 2008, and 2015), Fuhai (2000 and 2015), and Fukang (2007 and 2015).

\section{METHODS}

\section{Study population}

This study was conducted in accordance with the Declaration of Helsinki and with approval from the Ethics Committee of the People's Hospital of Xinjiang Uygur Autonomous Region. Results were based on data from seven independent crosssectional surveys performed in Hefeng (1998, 2008, and 2015), Fuhai (2000 and 2015), and Fukang (2007 and 2015). Methods were similar for all seven surveys: at least 2 days prior to the survey, all the inhabitants $\geq 30$ years of age in these specific area were informed of the survey contents, such as necessity of fasting overnight and physical examination. After signing the informed consent, subjects were enrolled in the study. While analyzing, the data collected in 1998 and 2000 were grouped as 1998-2000, the data collected in 2007 and 2008 as 2007-2008, and data collected in 2015 as the 2015 group. Of the 1,609 people who participated in 1998-2000, complete data were obtained from 1,551 subjects (96.4\%). Likewise, 2,331 (96.6\%) of 2,412 subjects in 2007-2008 and $2,262(93.3 \%)$ of 2,423 subjects in 2015 participated in the survey. We then excluded subjects with any missing data on lipid profiles and subjects who did not fast for at least $12 \mathrm{~h}$ before blood sampling. Finally, data on 1,059 subjects from 1998-2000, 2,144 subjects from 2007-2008, and 1,939 subjects from 2015 were analyzed.

\section{Data collection}

Data was collected during a single clinic visit with doctors or trained nurses using a standard questionnaire in face-to-face interviews. The questions assessed: demographic characteristics (age, sex, ethnicity and address), socioeconomic data (marital status and occupation), lifestyle risk factors (smoking and drinking status), and past medical history. Physical examination was conducted, including measurement of blood pressure, body height, and body weight. Blood pressure was presented as the mean of three measurements using a mercury sphygmomanometer via a standardized procedures recommended by the American Heart Association. ${ }^{27}$ All participants were advised to avoid cigarette smoking, alcohol, caffeinated beverages, tea, and exercise for at least $30 \mathrm{~min}$ prior to measurement. Three blood pressure measurements were taken after a rest of at least $5 \mathrm{~min}$, from the unclothed right arm of the person in a sitting position at intervals of at least $1 \mathrm{~min}$. Body weight, height, and WC were measured using standard methods. ${ }^{28}$ Height and Weight were measured to the nearest $0.1 \mathrm{~cm}$ and $0.1 \mathrm{~kg}$, respectively, with the participants in light-weight clothing and without shoes. Waist circumference (WC) was measured at the midpoint between the lower rib and upper margin of the iliac crest to the nearest $0.1 \mathrm{~cm}$ at the end of a normal expiration. Body mass index (BMI) was calculated by dividing weight by height squared $\left(\mathrm{kg} / \mathrm{m}^{2}\right)$.

\section{Laboratory measurements}

Venous blood was drawn from all participants who had fasted for $\geq 12 \mathrm{~h}$. Blood samples were obtained from median cubital vein into Vacutainer tubes without additives. Serum was separated immediately and stored at $-80^{\circ} \mathrm{C}$. All blood samples were measured within 1 month in the People's Hospital of Xinjiang Uygur Autonomous Region. TC, triglyceride (TG), HDL-C, LDL-C, and fasting blood glucose (FBG) were measured using enzymatic methods. Non-HDL-C was calculated as serum TC level minus HDL-C level.

\section{Diagnostic criteria}

According to the prevention standard proposed of dyslipidemia in China in $2016,{ }^{29}$ TC $\geq 6.2 \mathrm{mmol} / \mathrm{L} \quad(240 \mathrm{mg} / \mathrm{dL})$, TG $\geq 2.3 \mathrm{mmol} / \mathrm{L}(200 \mathrm{mg} / \mathrm{dL}), \mathrm{HDL}-\mathrm{C}<1.0 \mathrm{mmol} / \mathrm{L}(40 \mathrm{mg} / \mathrm{dL})$, and LDL-C $\geq 4.2 \mathrm{mmol} / \mathrm{L}(160 \mathrm{mg} / \mathrm{dL})$ were indicative of high TC, high TG, low HDL-C, and high LDL-C, respectively. Dyslipidemia was defined as high TC, and/or high TG, and/or low HDL-C, and/or high LDL-C, and/or having received during treatment for dyslipidemia in the last 2 weeks. Hypertension was defined as having a systolic blood pressure $\geq 140 \mathrm{~mm} \mathrm{Hg}$, and/or diastolic blood pressure $\geq 90 \mathrm{mmHg}$, and/or receiving antihypertensive medication. ${ }^{30}$ Overweight and obesity were defined according to the WHO classifications. ${ }^{31}$ BMI $\geq 25 \mathrm{~kg} / \mathrm{m}^{2}$ and BMI $\geq 30 \mathrm{~kg} / \mathrm{m}^{2}$ indicated overweight and obesity, respectively. Abdominal obesity was defined as having a WC $\geq 102 \mathrm{~cm}$ for men and $\geq 88 \mathrm{~cm}$ for women. ${ }^{31}$ Smokers were defined as those who smoked at least one cigarette per day and had lasted for at least 6 months. Drinkers were defined as those who drank at least twice per month (more than $640 \mathrm{~mL}$ beer or $100 \mathrm{~mL}$ Chinese liquor; about $57 \mathrm{~g}$ alcohol), and had lasted for at least 6 months.

\section{Statistical analysis}

The database was constructed with Excel 2007 Software (Microsoft Corp, Redmond, WA, USA) by two data managers and was corrected to guarantee the accuracy and integration of the data. The data were analyzed using SPSS 19.0 software (SPSS Inc., Chicago, IL, USA). Continuous variables were presented as means (standard deviations [SDs]) and were analyzed using $t$-test or ANOVA. Categorical variables were expressed as proportion $(\%)$ and frequency $(n)$ and were analyzed using the Chi-square test. In this analysis, the standardized population was determined using the total study population between 1998 and 2015. Age-standardized prevalence of dyslipidemia for each study period (1998-2000, 2007-2008, and 2015) was then calculated according to the standardized population. As for dyslipidemia, adjusted odds ratios (ORs) with associated 95\% confidence intervals (CIs) were calculated using multivariate logistic regression model through the Enter method. All the study 
population between 1998 and 2015 was included in multivariate logistic regression model. Covariates were gender, age, ethnicity, smoking, drinking, BMI, WC, hypertension, and FBG in the models. All statistical tests were two-sided and $P$-values $<0.05$ were considered significant.

\section{RESULTS}

\section{Population characteristics}

Table 1 details the characteristics of the study participants. The proportion of male was $41.5 \%$ in $1998-2000$, $40.8 \%$ in $2007-$ 2008 , and $45.5 \%$ in 2015. Mean age of the subjects was 48.5 (SD, 11.6) years, 47.9 (SD, 10.9) years, and 53.7 ( $\mathrm{SD}, 13.3$ ) years, respectively. There were differences in ethnic composition among the three groups. The proportion of smokers declined across the survey years, whereas drinking status showed no difference between the survey years. Mean BMI was significantly higher in 2007-2008 populations than in 1998-2000 and 2015 populations, but there were no significant differences observed in the mean level of WC and FBG.

\section{Trends in lipid profiles}

Table 2 shows the trends in lipid profiles. Mean LDL-C decreased from 3.53 (SD, 1.09) $\mathrm{mmol} / \mathrm{L}$ in 1998-2000 to 2.67 $(\mathrm{SD}, 0.78) \mathrm{mmol} / \mathrm{L}$ in $2015(P<0.001)$. Mean TC was 4.79 (SD, $1.15) \mathrm{mmol} / \mathrm{L}$ in $1998-2000,5.17$ (SD, 1.04) $\mathrm{mmol} / \mathrm{L}$ in $2007-$ 2008, and 4.59 (SD, 0.95) mmol/L in 2015. Mean TG was 0.94 $(\mathrm{SD}, 0.57) \mathrm{mmol} / \mathrm{L}$ in $1998-2000,1.58(\mathrm{SD}, 1.28) \mathrm{mmol} / \mathrm{L}$ in 2007-2008, and 1.11 (SD, 0.87) mmol/L in 2015. Mean HDL-C was $1.08(\mathrm{SD}, 0.36) \mathrm{mmol} / \mathrm{L}$ in $1998-2000,1.50(\mathrm{SD}, 0.50)$ $\mathrm{mmol} / \mathrm{L}$ in 2007-2008, and 1.44 (SD, 0.33) $\mathrm{mmol} / \mathrm{L}$ in 2015. Mean non-HDL-C was 3.71 (SD, 1.13) mmol/L in 1998-2000, $3.68(\mathrm{SD}, 1.00) \mathrm{mmol} / \mathrm{L}$ in $2007-2008$, and $3.16(\mathrm{SD}, 0.90)$ $\mathrm{mmol} / \mathrm{L}$ in 2015. As for lipid ratios, the ratios of TC/HDL-C and LDL-C/HDL-C showed decreasing trends from 1998 to 2015 $(P<0.001)$. Mean TG/HDL-C ratios was 0.99 (SD, 0.76) in 1998-2000, 1.20 (SD, 1.06) in 2007-2008, and 0.93 (SD, 0.87) in 2015 .

Table 1. Demographic characteristics of subjects, 1998-2015

\begin{tabular}{lcrrr}
\hline Parameters & $\begin{array}{r}1998-2000 \\
(n=1,059)\end{array}$ & $\begin{array}{r}2007-2008 \\
(n=2,144)\end{array}$ & $\begin{array}{c}2015 \\
(n=1,939)\end{array}$ & $P$ \\
\hline Gender & & & & \\
$\quad$ Male, $n(\%)$ & $439(41.5)$ & $874(40.8)$ & $882(45.5)$ & 0.006 \\
$\quad$ Female, $n(\%)$ & $620(58.5)$ & $1270(59.2)$ & $1057(54.5)$ & \\
Age, years & $48.5(11.6)$ & $47.9(10.9)$ & $53.7(13.3)$ & $<0.001$ \\
30-39, $n(\%)$ & $243(22.9)$ & $540(25.2)$ & $282(14.5)$ & \\
$40-49, n(\%)$ & $361(34.1)$ & $730(34.0)$ & $594(30.6)$ & \\
$50-59, n(\%)$ & $251(23.7)$ & $528(24.6)$ & $440(22.7)$ & \\
$\geq 60, n(\%)$ & $204(19.3)$ & $346(16.2)$ & $623(32.2)$ & \\
Ethnicity & & & & \\
Han, $n(\%)$ & $63(5.9)$ & $454(21.2)$ & $1158(59.7)$ & \\
$\quad$ Mongolian, $n(\%)$ & $318(30.0)$ & $496(23.1)$ & $386(19.9)$ & $<0.001$ \\
Kazakh, $n(\%)$ & $678(64.1)$ & $1194(55.7)$ & $395(20.4)$ & \\
Smoking, $n$ (\%) & $267(25.2)$ & $503(23.5)$ & $414(21.4)$ & 0.046 \\
Drinking, $n$ (\%) & $234(22.1)$ & $462(21.5)$ & $427(22.0)$ & 0.912 \\
BMI, kg/m ${ }^{2}$, mean (SD) & $25.4(4.3)$ & $26.7(4.3)$ & $26.1(4.2)$ & $<0.001$ \\
WC, cm, mean (SD) & - & $85.3(11.4)$ & $90.0(11.6)$ & 0.806 \\
SBP, mm Hg, mean (SD) & $148.3(31.7)$ & $134.1(24.9)$ & $128.6(20.6)$ & $<0.001$ \\
DBP, mm Hg, mean (SD) & $91.8(18.1)$ & $86.1(14.1)$ & $76.4(11.7)$ & $<0.001$ \\
FBG, mmol/L, mean (SD) & - & $5.32(1.24)$ & $5.17(1.30)$ & 0.481 \\
\hline
\end{tabular}

BMI, body mass index; DBP, diastolic blood pressure; FBG, fasting blood glucose; SBP, systolic blood pressure; SD, standard deviation; WC, waist circumference.
Table 2. Serum lipid and lipoprotein levels in subjects, 19982015

\begin{tabular}{lcccc}
\hline & $1998-2000$ & $2007-2008$ & 2015 & $P$ value \\
\hline TC, mmol/L & $4.79(1.15)$ & $5.17(1.04)$ & $4.59(0.95)$ & $<0.001$ \\
TG, mmol/L & $0.94(0.57)$ & $1.58(1.28)$ & $1.11(0.87)$ & $<0.001$ \\
HDL-C, mmol/L & $1.08(0.36)$ & $1.50(0.50)$ & $1.44(0.33)$ & $<0.001$ \\
LDL-C, mmol/L & $3.53(1.09)$ & $2.87(1.05)$ & $2.67(0.78)$ & $<0.001$ \\
Non-HDL-C, & $3.71(1.13)$ & $3.67(1.02)$ & $3.15(0.90)$ & $<0.001$ \\
mmol/L & $5.00(3.61)$ & $3.72(1.21)$ & $3.31(0.85)$ & $<0.001$ \\
TC/HDL-C & $1.01(0.76)$ & $1.20(1.06)$ & $0.93(0.87)$ & $<0.001$ \\
TG/HDL-C & $3.80(2.06)$ & $2.06(0.87)$ & $1.93(0.65)$ & $<0.001$ \\
LDL-C/HDL-C & 3.801
\end{tabular}

HDL-C, high-density lipoprotein cholesterol; LDL-C, low-density lipoprotein cholesterol; TC, total cholesterol; TG, triglyceride.

Data are presented as mean (standard deviation).

Table 3. Trends in prevalence of dyslipidemia, 1998-2015

\begin{tabular}{lclc}
\hline & \multicolumn{1}{c}{$1998-2000$} & \multicolumn{1}{c}{$2007-2008$} & \multicolumn{1}{c}{2015} \\
\hline High TC & $9.3(7.5-11.0)$ & $14.9(13.4-16.4)$ & $5.2(4.3-6.3)$ \\
High TG & $3.2(2.1-4.3)$ & $20.1(18.4-21.8)$ & $6.9(6.0-8.2)$ \\
Low HDL-C & $40.4(37.5-43.7)$ & $10.2(8.9-11.4)$ & $6.8(5.8-8.0)$ \\
High LDL-C & $24.6(22.0-27.1)$ & $12.2(10.8-13.6)$ & $4.6(3.8-5.6)$ \\
Dyslipidemia & $53.5(50.5-56.5)$ & $38.6(36.6-40.6)$ & $16.8(15.3-18.7)$ \\
\hline
\end{tabular}

HDL-C, high-density lipoprotein cholesterol; LDL-C, low-density lipoprotein cholesterol; TC, total cholesterol; TG, triglyceride.

Data are presented as prevalence ( $95 \%$ confidence interval).

\section{Trends in the prevalence of dyslipidemia}

The trends in the prevalence of dyslipidemia among participants are presented in Table 3. From 1998 to 2015, the prevalence of dyslipidemia decreased from $53.5 \%$ in $1998-2000$ to $38.6 \%$ in 2007-2008 and to $16.8 \%$ in 2015. The prevalence of low HDL-C and high LDL-C also showed the same trends across the survey years. Prevalence of high TC was 9.3\% in 1998-2000, $14.9 \%$ in 2007-2008, and 5.2\% in 2015. The prevalence of high TG was $3.2 \%$ in $1998-2000,20.1 \%$ in $2007-2008$, and $6.9 \%$ in 2015, respectively. Figure 1 shows trends in age-standardized prevalence of dyslipidemia. The age-standardized prevalence of dyslipidemia, high TC, high TG, low HDL-C, and high LDL-C was $53.6 \%, 9.2 \%, 3.2 \%, 40.3 \%$, and $24.6 \%$ in $1998-2000,26.5 \%$, $15.7 \%, 20.7 \%, 9.8 \%$, and $12.8 \%$ in $2007-2008$, and $16.9 \%, 5.0 \%$, $7.1 \%, 7.2 \%$, and $4.4 \%$ in 2015 , respectively. The prevalence of the clustering of 1,2 , and $\geq 3$ kinds of dyslipidemia among participants showed significantly declining trends, as seen in Figure 2.

\section{Factors associated with dyslipidemia}

As shown in Table 4, multivariate logistic regression analysis suggested that the prevalence of dyslipidemia was closely related with male gender, Mongolian ethnicity, hypertension, obesity, elevated FBG, smoking, and drinking. Table 5 shows the factors associated with high TC, high TG, low HDL-C, and high LDL-C among the participants. The prevalence of high TG, low HDL-C, and high LDL-C were related with BMI (OR 1.574; 95\% CI, 1.361-1.822, OR 1.273; 95\% CI, 1.071-1.514, and OR 1.523; 95\% CI, 1.284-1.807, respectively). Abdominal obesity was a risk factor only for high TG (OR 1.515; 95\% CI, 1.158-1.982). Hypertension increased the prevalence of high TC (OR 1.355; 95\% CI, 1.091-1.683) and high TG (OR 1.706; 95\% CI, 


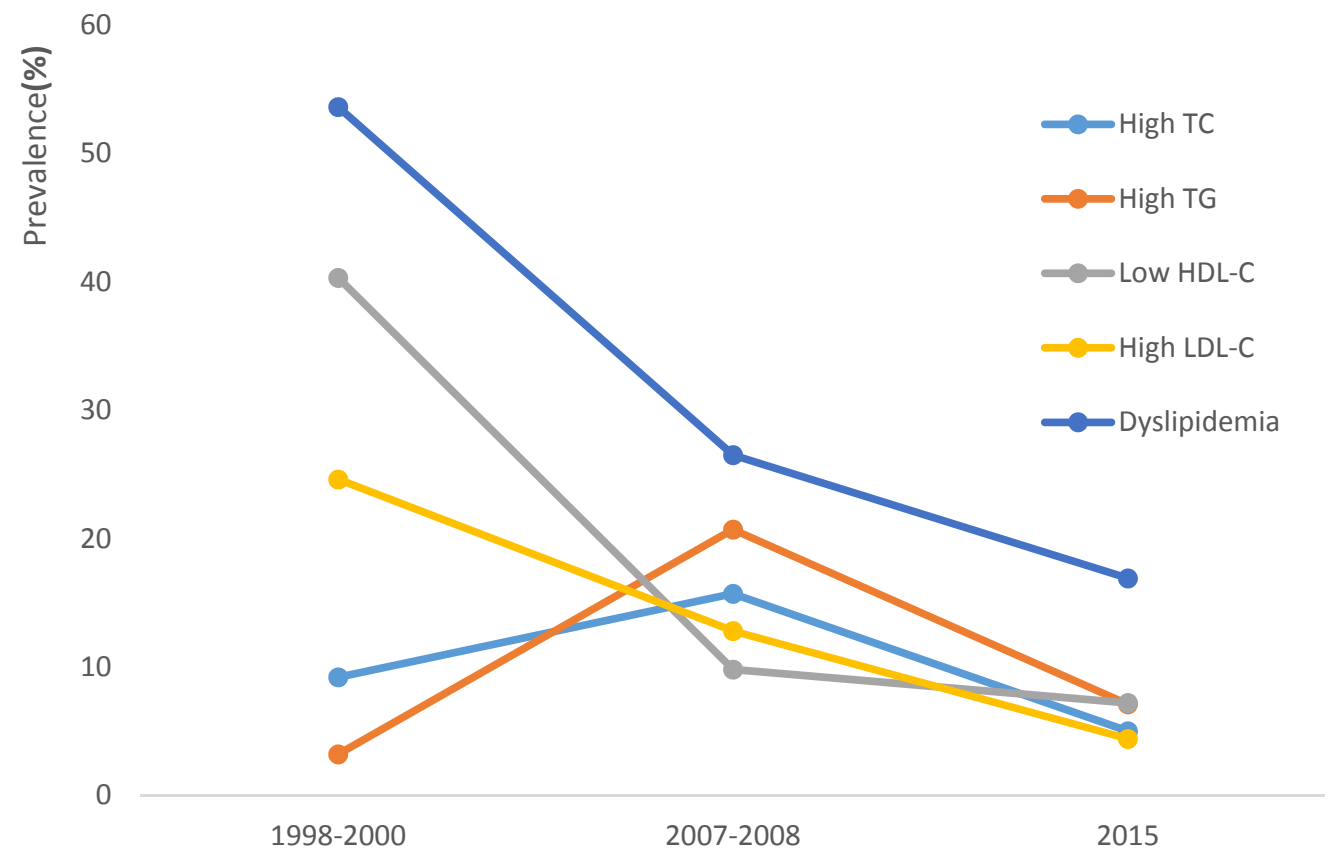

Figure 1. Trends in age-standardized prevalence of high TC, high TG, low LDL-C, high HDL-C, and dyslipidemia from 1998-2015

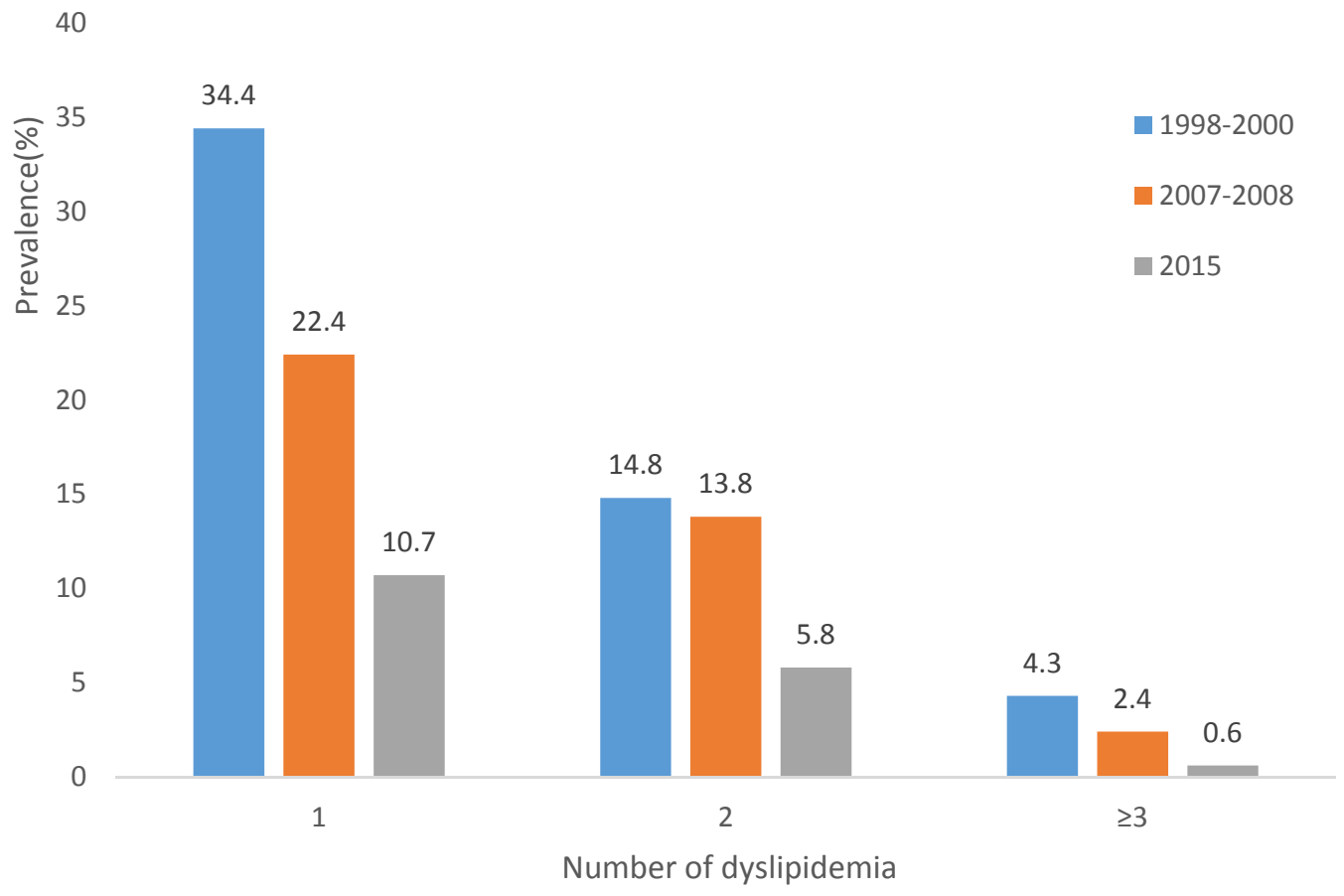

Figure 2. Trends in prevalence of the clustering of dyslipidemia

1.403-2.075). The prevalence of high TC, high TG, low HDL-C, and high LDL-C were closely related with FBG (OR 2.362; 95\% CI, 1.573-3.545, OR 2.499; 95\% CI, 1.740-3.589, OR 2.042; 95\% CI, 1.280-3.257, and OR 2.037; 95\% CI, 1.299-3.193, respectively). With regard to smoking and alcohol consumption, we observed an association between smoking and the prevalence of high TC, high TG, and high LDL-C (OR 1.666; 95\% CI, 1.223-2.269, OR 1.328; 95\% CI, 1.019-1.731, and OR 1.489; 95\% CI, 1.081-2.051, respectively).

\section{DISCUSSION}

This is the first report on secular trends in serum lipids in low income northwestern Xinjiang Province, mainly comprised of Han, Kazakh, and Mongolian ethnicities. Our study has found that there was a declining trend in the prevalence of dyslipidemia from 1998-2000 through 2007-2008 and to 2015. There are several reasons for the changes of epidemiological characteristics of dyslipidemia in northwestern Xinjiang. First of all, with the improvement of economic and education level, health awareness 
Table 4. Factors associated with the prevalence of dyslipidemia from multivariate logistic regression

\begin{tabular}{llllcl}
\hline Variables & Stratification & $\beta$ & Wald & OR (95\% CI) & $P$ value \\
\hline Gender & Female & 1 & & & \\
Age, years & Male & 0.219 & 5.304 & $1.245(1.033-1.501)$ & 0.021 \\
& $30-39$ & 1 & & & \\
& $40-49$ & -0.036 & 0.120 & $0.965(0.788-1.182)$ & 0.730 \\
& $50-59$ & 0.054 & 0.244 & $1.056(0.081-1.311)$ & 0.622 \\
Ethnicity & $\geq 60$ & -0.097 & 0.700 & $0.907(0.722-1.140)$ & 0.403 \\
& Han & 1 & & & \\
Body mass index & Mongolian & 0.200 & 4.149 & $1.221(1.008-1.479)$ & 0.042 \\
& Kazakh & -0.171 & 3.838 & $0.843(0.710-1.000)$ & 0.050 \\
& Normal & 1 & & & \\
Abdominal obesity & Overweight & 0.423 & 26.093 & $1.527(1.298-1.797)$ & $<0.001$ \\
& Obesity & 0.653 & 31.723 & $1.922(1.531-2.413)$ & $<0.001$ \\
Hypertension & Yes & 1 & & & \\
FBG, mmol/L & No & 0.254 & 5.047 & $1.289(1.033-1.609)$ & 0.025 \\
& Yes & 1 & & & \\
Smoking & $<7.0$ & 1 & & & \\
& $\geq 7.0$ & 0.907 & 29.228 & $2.477(1.783-3.442)$ & $<0.001$ \\
Drinking & No & 1 & & & \\
& Yes & 0.255 & 6.145 & $1.290(1.055-1.579)$ & 0.013 \\
& No & 1 & & & \\
\hline & Yes & 0.279 & 6.932 & $1.322(1.074-1.628)$ & 0.008 \\
\hline
\end{tabular}

CI, confidence interval; FBG, fasting blood glucose.

Table 5. Factors associated with the prevalence of lipid parameters abnormality

\begin{tabular}{|c|c|c|c|c|c|}
\hline Lipid parameters & Factors & $\beta$ & Wald & OR $(95 \% \mathrm{CI})$ & $P$ value \\
\hline \multirow[t]{5}{*}{ High TC } & Age & 0.307 & 33.057 & $1.359(1.224-1.509)$ & $<0.001$ \\
\hline & Ethnicity & 0.339 & 28.306 & $1.403(1.239-1.590)$ & $<0.001$ \\
\hline & Hypertension & 0.304 & 7.561 & $1.355(1.091-1.683)$ & 0.006 \\
\hline & Fasting blood glucose & 0.859 & 17.202 & $2.362(1.573-3.545)$ & $<0.001$ \\
\hline & Smoking & 0.511 & 10.494 & $1.666(1.223-2.269)$ & 0.001 \\
\hline \multirow[t]{8}{*}{ High TG } & Age & -0.125 & 6.955 & $0.883(0.804-0.968)$ & 0.008 \\
\hline & Ethnicity & -0.475 & 64.873 & $0.622(0.554-0.698)$ & $<0.001$ \\
\hline & Hypertension & 0.534 & 28.680 & $1.706(1.403-2.075)$ & $<0.001$ \\
\hline & Body mass index & 0.454 & 37.198 & $1.574(1.361-1.822)$ & $<0.001$ \\
\hline & Abdominal obesity & 0.415 & 9.172 & $1.515(1.158-1.982)$ & 0.002 \\
\hline & Fasting blood gluco & 0.916 & 24.589 & $2.499(1.740-3.589)$ & $<0.001$ \\
\hline & Smoking & 0.284 & 4.413 & $1.328(1.019-1.731)$ & 0.036 \\
\hline & Drinking & 0.347 & 7.242 & $1.416(1.099-1.823)$ & 0.007 \\
\hline \multirow[t]{4}{*}{ Low HDL-C } & Gender & 0.364 & 5.903 & $1.438(1.073-1.929)$ & 0.015 \\
\hline & Age & -0.260 & 20.131 & $0.771(0.689-0.864)$ & $<0.001$ \\
\hline & Body mass index & 0.241 & 7.461 & $1.273(1.071-1.514)$ & 0.006 \\
\hline & Fasting blood glucose & 0.714 & 8.977 & $2.042(1.280-3.257)$ & 0.003 \\
\hline \multirow[t]{6}{*}{ High LDL-C } & Gender & 0.309 & 4.278 & $1.362(1.016-1.825)$ & 0.039 \\
\hline & Age & 0.235 & 16.590 & $1.265(1.130-1.416)$ & $<0.001$ \\
\hline & Ethnicity & 0.291 & 17.723 & $1.338(1.168-1.532)$ & $<0.001$ \\
\hline & Body mass index & 0.421 & 23.281 & $1.523(1.284-1.807)$ & $<0.001$ \\
\hline & FBG & 0.711 & 9.607 & $2.037(1.299-3.193)$ & 0.002 \\
\hline & Fasting blood glucose & 0.398 & 5.942 & $1.489(1.081-2.051)$ & 0.015 \\
\hline
\end{tabular}

HDL-C, high-density lipoprotein cholesterol; LDL-C, low-density lipoprotein cholesterol; TC, total cholesterol; TG, triglyceride.

has been improved. ${ }^{23-25}$ Moreover, the Chinese government has increased its investment in medical and health services in recent years. ${ }^{26}$ In addition, since the establishment in 1997, our center has been focusing on the population-based prevention of CVD in Xingjiang by training local health workers. Furthermore, in 2009, the Chinese government launched the construction of nomads settlement, and 110.5 thousand nomadic families were supported by the government, which brought about higher education attainment and health awareness improvement, followed by healthy lifestyles and eating habits, such as more intake of fresh vegetables and fruits. Furthermore, nationwide data suggest that patients with dyslipidemia showed an improvement in lipid lowering treatment and in achieving therapeutic goals in LDL-C over the past decade. For example, $61.5 \%$ of very high and high risk patients with lipid-lowering agents in 2012 achieved the therapeutic goal of LDL-C, compared to only $16.6 \%$ in $2000 .{ }^{32,33}$ A similar trend of lipids was found in the developed countries. Declines in mean TC and LDL-C have been recently noted in high-income regions, such as Australasia, North America, and Western Europe. ${ }^{13,34,35}$ Although the mean LDL-C was still higher in the United States than in China between 1988 and 2010, the mean TC, TG, LDL-C, and non-HDL-C levels decreased and the mean HDL-C level increased linearly. ${ }^{36}$ Among Chinese adults, the prevalence of dyslipidemia has been increasing during the past decade. Statistics from The National Nutrition and Health Survey in 2002 reported that the prevalence of high TC, high TG, and low HDL-C was $2.9 \%, 11.9 \%$, and $7.4 \%$, respectively. ${ }^{14}$ In 2012, a survey of adults in China showed that the prevalence of high TC, high TG, and low HDL-C was 4.9\%, 13.1\%, and 33.9\%, respectively. ${ }^{15}$ The increased prevalence of dyslipidemia may be related to dietary pattern changes (such as a higher intake of fat and reduced fiber intake and lower physical activity levels).

We found that the prevalence of dyslipidemia was closely related with male gender, Mongolian ethnicity, hypertension, obesity, elevated FBG, smoking, and drinking in multivariate logistic regression analysis. Compared with females, males had greater odds of having dyslipidemia (OR 1.245), which is in accordance with most previous studies. ${ }^{37,38}$ However, one study found that dyslipidemia was more prevalent in women. ${ }^{39}$ Our study demonstrated that the prevalence of low HDL-C was higher in men, similar to the result of previous surveys. ${ }^{40}$ In this study, we found that the prevalence of high TC and LDL-C closely related with age. A similar trend of lipid distribution was found in previous studies. ${ }^{37,41}$ In our study, the risk of dyslipidemia in Mongolian participants was 1.221 times higher than in Han participants. With regard to the differences across ethnic groups in the prevalence of dyslipidemia, the mechanisms are not clear. Most Kazakh and Mongolian live as herders and reside in the pastoral areas north of Xinjiang. In addition, Kazakh and Mongolian share similar dietary habits, characterized by eating more animal fat and consuming less grain. ${ }^{42}$ Kazakh ethnicity is mainly distributed in Kazakhstan, China, Russia, Uzbekistan, Turkey, and Mongolia, while the Mongolians are mainly distributed in China, Mongolia, and Russia. Therefore, the current study may provide a theoretical basis for the prevention and treatment of dyslipidemia in the two ethnicities, which share common lifestyles.

Moreover, other environmental factors, such as cigarette smoking, alcohol consumption, and physical inactivity, are determinants of HDL-C level. Smoking and drinking can decrease the HDL-C levels. ${ }^{43}$ Increased physical activity is known to be related to higher HDL-C level. ${ }^{44}$ The relationship between dyslipidemia and conventional factors, including BMI, WC, BP, and FBG, were also analyzed. Prevalence of high TG, high LDL-C, and low HDL-C was all closely associated with BMI, in agreement with those of previous studies. ${ }^{37,44}$ Dyslipidemia is more prevalent in abdominal obesity. However, WC was a risk factor only for high TG after adjusting for other factors, possible due to the fact that the participants in this study have less WC and the effect on lipid is corrected by co-existing factors. Hypertension increased the prevalence of high TC and high TG, in accordance with existing evidence. ${ }^{38,45}$ In addition, all kinds of dyslipidemia were closely associated with FBG. 
The present study also has several limitations. First, there were differences in population composition regarding age, gender, and ethnicity among the three groups, which might have brought about biased information. Second, we did not have information available on our participants regarding participation in dietary factors, family income, and other factors that increase the risk of developing dyslipidemia. In addition, important information that affects lipid levels and can explain the lowering prevalence and lipid levels, like use of lipid lowering medicine and physical activity, were not included in the analysis, which made some of conclusions based on speculations. Finally, this study was limited to population from three counties of Northwestern Xinjiang, where a long-term health provider education program of CVD risk factors, especially hypertension, has been implemented since 1997; therefore, the results may not be representative for the whole of Xinjiang Province.

In conclusion, there was a decrease in the prevalence of dyslipidemia among adults of northwestern Xinjiang from 1998 through 2015. In addition, we also examined various risk factors for dyslipidemia. Our results are expected to be helpful in developing appropriate prevention and control strategies for these modifiable risk factors of dyslipidemia and decrease the overall CVD mortality and morbidity.

\section{ACKNOWLEDGMENTS}

We thank all the study participants for supporting our job and thank volunteers for providing great help. We also thank leaders of health bureaus, Mr Zou Bo at Fukang, Mr Chu Xiangdong at Hefeng county and Mr Jin Yihai at Fuhai for their support and for cooradinating their efforts and thank leaders of county hospitals, Mr Wu Junjiang at Fukang, Mr Zhao Jianxin at Hefeng and Mr Yuan Wangyan at Fuhai for the their greatest support and on-site overseeing the survey process and helping by regulating their human resource. Finally, we thank the Ministry of Science and Technology for funding the project.

Author contribution: Nanfang Li and Ling Zhou put forward, designed, and implemented the study, and analyzed the data, and drafted the manuscript. Xin Zhao, Mulalibieke Heizhati, Suofeiya Abulikemu, Delian Zhang, Qiuyan Cheng, Weijin Ouyang, Xiaoguang Yao, Jing Hong, Ting Wu, Zuhere Xiamili and Nanfang $\mathrm{Li}$ participated in the investigated design and implementation, data analysis and the manuscript writing.

Declaration of interest and source of funding: This study was funded by the Ministry of Science and Technology of the People's Republic of China [grant number 2013GS650101].

Conflicts of interest: None declared.

\section{REFERENCES}

1. World Health Organization. Cardiovascular diseases (CVDs). http:// www.who.int/mediacentre/factsheets/fs317/en/; 2017 Accessed 10.07.17.

2. Wilson PWD, D'Agostino RB, Levy D, et al. Prediction of coronary heart disease using risk factor categories. Circulation. 1998;97(18): 1837-1847.

3. Lipid Research Clinics Program. The Lipid Research Clinics Coronary Primary Prevention Trial results. I: reduction in incidence of coronary heart disease. JAMA. 1984;251(3):351-364.

4. Lipid Research Clinics Program. The Lipid Research Clinics Coronary Primary Prevention Trial results, II: the relationship of reduction in incidence of coronary heart disease to cholesterol lowering. JAMA. 1984;251(3):365-374.

5. Stamler J, Wentworth D, Neaton JD. Is relationship between serum cholesterol and risk of premature death from coronary heart disease continuous and graded? Findings in 356,222 primary screenees of the Multiple Risk Factor Intervention Trial (MRFIT). JAMA. 1986; 256(20):2823-2828.

6. Wilson PW, Garrison RJ, Castelli WP, et al. Prevalence of coronary heart disease in the Framingham Offspring Study: role of lipoprotein cholesterols. Am J Cardiol. 1980;46(4):649-654.

7. Assmann G, Schulte H, von Eckardstein A, et al. High-density lipoprotein cholesterol as a predictor of coronary heart disease risk: the PROCAM experience and pathophysiological implications for reverse cholesterol transport. Atherosclerosis. 1996;124(suppl):S11S20.

8. Baigent C, Keech A, Kearney PM, et al; Cholesterol Treatment Trialists' (CTT) Collaborators. Efficacy and safety of cholesterollowering treatment: prospective meta-analysis of data from 90,056 participants in 14 randomised trials of statins. Lancet. 2005; 366(9493):1267-1278.

9. Toth PP. Subclinical atherosclerosis: what it is, what it means and what we can do about it. Int J Clin Pract. 2008;62(8):1246-1254.

10. Grover SA, Abrahamowicz M, Joseph L, et al. The benefits of treating hyperlipidemia to prevent coronary heart disease. Estimating changes in life expectancy and morbidity. JAMA. 1992;267(6):816822 .

11. Okamura T. Dyslipidemia and cardiovascular disease: a series of epidemiologic studies in Japanese populations. J Epidemiol. 2010; 20(4):259-265.

12. Murray CJ, Lopez AD. Mortality by cause for eight regions of the world: Global Burden of Disease Study. Lancet. 1997;349(9061): 1269-1276.

13. Farzadfar F, Finucane MM, Danaei G, et al; Global Burden of Metabolic Risk Factors of Chronic Diseases Collaborating Group (Cholesterol). National, regional, and global trends in serum total cholesterol since 1980: systematic analysis of health examination surveys and epidemiological studies with 321 country-years and 3.0 million participants. Lancet. 2011;377(9765):578-586.

14. Zhao WH, Zhang J, Zhai Y, et al. Blood lipid profile and prevalence of dyslipidemia in Chinese adults. Biomed Environ Sci. 2007;20: 329-335.

15. Department of Disease Control and Prevention of National Bureau of health and Family Planning Commission of China. Report on nutrition and chronic diseases in Chinese Residents (2015). Beijing: People's Health Publishing House; 2015.

16. Moran A, Gu D, Zhao D, et al. Future cardiovascular disease in China: markov model and risk factor scenario projections from the coronary heart disease policy model-China. Circ Cardiovasc Qual Outcomes. 2010;3(3):243-252.

17. Guo SX, Ma RL, Guo H, et al. Epidemiological analysis of dyslipidemia in adults of three ethnicities in Xinjiang, China. Genet Mol Res. 2014;13(2):2385-2393.

18. Zhou J, Yao H, Wang HC, et al. Epidemic features of dyslipidemia among Uygur, Kazakh and Han ethnic groups in Urumqi. Zhonghua Yi Xue Za Zhi. 2016;96(38):3090-3093.

19. Yao XG, Frommlet F, Zhou L, et al. The prevalence of hypertension, obesity and dyslipidemia in individuals of over 30 years of age belonging to minorities from the pasture area of Xinjiang. BMC Public Health. 2010;10:91.

20. Li N, Wang H, Yan Z, Yao X, Hong J, Zhou L. Ethnic disparities in the clustering of risk factors for cardiovascular disease among the Kazakh, Uygur, Mongolian and Han populations of Xinjiang: a cross-sectional study. BMC Public Health. 2012;12:499.

21. Li LL, Zhang YM, Du JY, et al. Analysis of metabolic features and food composition related with pathogenesis of type 2 diabetes mellitus in population of Uygurs and Kazaks in Xinjiang. Zhonghua Neifenmi Daixie Zazhi. 2005;21:141-142.

22. Yan WL, Yang XY, Zheng YJ, et al. The Metabolic Syndrome in Uygur and Kazak Populations. Diabetes Care. 2005;28(10):25542555.

23. National Bureau of Statistics of China. The 2000 population census 
of the People's Republic of China. http://www.stats.gov.cn/tjsi/ $\mathrm{pcsj} / \mathrm{rkpc} / 5 \mathrm{rp} /$ index.htm; 2002. Accessed 12.12.02.

24. National Bureau of Statistics of China. China Statistical Yearbook 2016. http://www.stats.gov.cn/tjsj/ndsj/2016/indexch.htm; 2016. Accessed 12.07.17.

25. National Bureau of Statistics of China. The 2010 population census of the People's Republic of China. http://www.stats.gov.cn/tjsj/ $\mathrm{pcsj} / \mathrm{rkpc} / 6 \mathrm{rp} /$ indexch.htm; 2012. Accessed 20.06.12.

26. China National Center for Cardiovascular Diseases. Report on cardiovascular disease in China 2012. Beijing: Encyclopedia of China Publishing House; 2013.

27. Perloff D, Grim C, Flack J, et al. Human blood pressure determination by sphygmomanometry. Circulation. 1993;88:2460-2470.

28. World Health Organ. Obesity: Preventing and Managing the Global Epidemic. Geneva, Switzerland: WHO; 2000; Volume 894, pp. 1253.

29. Joint Committee for the Prevention and Treatment of Dyslipidemia in Chinese Adults. Guidelines for the prevention and treatment of dyslipidemia in Chinese adults (2016 Revised Edition). Chin J Cardiol. 2016:44(10):833-850.

30. Chobanian AV, Bakris GL, Black HR, et al; Joint National Committee on Prevention, Detection, Evaluation, and Treatment of High Blood Pressure. National Heart, Lung, and Blood Institute; National High Blood Pressure Education Program Coordinating Committee. Seventh report of the Joint National Committee on Prevention, Detection, Evaluation, and Treatment of High Blood Pressure. Hypertension. 2003;42(6):1206-1252.

31. Gurr MI. Diet, Nutrition and the Prevention of Chronic Diseases. Geneva, Switzerland: World Health Organization; 2003.

32. Zhao S, Wang Y, Mu Y, et al; DYSIS-China Study Investigators. Prevalence of dyslipidaemia in patients treated with lipid-lowering agents in China: results of the DYSlipidemia International Study (DYSIS). Atherosclerosis. 2014;235(2):463-469.

33. Current Status in Clinical Control of Hypercholesterolemia in China Research Group. A multi-center study of current status on clinical control of hypercholesterolemia in China: success rate and related factors. Zhonghua Xin Xue Guan Bing Za Zhi. 2002;30(2):109-114.
34. Ferrières $\mathrm{J}$, Bongard $\mathrm{V}$, Dallongeville $\mathrm{J}$, et al. Trends in plasma lipids, lipoproteins and dyslipidaemias in French adults, 1996-2007. Arch Cardiovasc Dis. 2009;102(4):293-301.

35. Samaranayaka S, Gulliford MC. Trends in cardiovascular risk factors among people with diabetes in a population based study, Health Survey for England 1994-2009. Prim Care Diabetes. 2013; 7:193-198.

36. Carroll MD, Kit BK, Lacher DA, et al. Trends in lipids and lipoproteins in US adults, 1988-2010. JAMA. 2012;308(15):15451554.

37. Erem C, Hacihasanoglu A, Deger O, et al. Prevalence of dyslipidemia and associated risk factors among Turkish adults: Trabzon lipid study. Endocrine. 2008;34:36-51.

38. Cai L, Zhang L, Liu A, et al. Prevalence, awareness, treatment, and control of dyslipidemia among adults Beijing, China. J Atheroscler Thromb. 2012;19(2):159-168.

39. Wang S, Xu L, Jonas JB, et al. Prevalence and associated factors of dyslipidemia in the adult Chinese population. PLoS One. 2011;6: e17326.

40. Li JH, Wang LM, Li YC, et al. Epidemiologic characteristics of dyslipidemia in Chinese adults in 2010. Chin J Prev Med. 2012; 46(5):414-418.

41. Sun GZ, Li Z, Guo L, et al. High prevalence of dyslipidemia and associated risk factors among rural Chinese adults. Lipids Health Dis. 2014;13:189.

42. Zhai F, He Y, Wang Z, et al. Status and characteristic of dietary intake of 12 minority nationalities in China. Wei Sheng Yan Jiu. 2007;36(5):539-541

43. Mureddu GF, Brandimarte F, De Luca L. High-density lipoprotein levels and risk of cardiovascular events: a review. J Cardiovasc Med (Hagerstown). 2012;13(9):575-586.

44. Wang B, Wei D, Wang C, et al. Prevalence of dyslipidemia and associated factors in the Yi farmers and migrants of Southwestern China. Atherosclerosis. 2012;223:512-518.

45. Khader YS, Batieha A, El-Khateeb M, et al. Prevalence of dyslipidemia and its associated factors among Jordanian adults. J Clin Lipidol. 2010;4:53-58. 